\title{
Finite Energy One-half Monopole Solutions of the SU(2) Yang-Mills-Higgs Theory.
}

\author{
Rosy Teh ${ }^{* *}$ \\ School of Physics, Universiti Sains Malaysia \\ 11800 USM Penang, Malaysia \\ E-mail: rosyteh@usm.my \\ Ban-Loong $\mathrm{Ng}$ and Khai-Ming Wong \\ School of Physics, Universiti Sains Malaysia \\ 11800 USM Penang, Malaysia \\ E-mail: banloong •ng@gmail . com, kmwong@usm.my
}

\begin{abstract}
We present finite energy SU(2) Yang-Mills-Higgs particles of one-half topological charge. The magnetic fields of these solutions at spatial infinity correspond to the magnetic field of a positive one-half magnetic monopole at the origin and a semi-infinite Dirac string on one-half of the $z$-axis carrying a magnetic flux of $\frac{2 \pi}{g}$ going into the origin. Hence the net magnetic charge is zero. The gauge potentials are singular along one-half of the $z$-axis, elsewhere they are regular.
\end{abstract}

PACS : $14.80 . \mathrm{HV}, 11.10 . \mathrm{Lm}$

36th International Conference on High Energy Physics

4-11 July 2012

Melbourne, Australia

\footnotetext{
* Speaker.

† Corresponding author.
} 


\section{Introduction}

The $3+1$ dimensional SU(2) Yang-Mills-Higgs (YMH) field theory, with the Higgs field in the adjoint representation possess magnetic monopole configurations [1]-[3]. The 't Hooft-Polyakov monopole solution of unit magnetic charge is spherically symmetric and possesses finite energy [1]. However, finite energy monopole configurations with either a magnetic charge greater than one [1] or possessing more than one pole cannot be spherical symmetric. They can however possess axial symmetry. Exact monopole solutions are known only in the Bogomol'nyi-PrasadSommerfield (BPS) limit [2]-[3]. Outside this limit, only numerical solutions are known. Numerical finite energy monopole solutions include the monopole-antimonopole-pair (MAP), monopoleantimonopole-chain (MAC), and vortex ring solutions [4] and they possess axial symmetry.

Recently we have constructed some exact singular one-half monopole configurations which are axially symmetric and possess a Dirac string along one-half of the $z$-axis [5]. Harikumar et al. [6] demonstrated the existence of generic smooth YM potentials of one-half monopoles but no exact or numerical solutions are given. In this paper, we discuss numerical, finite energy monopole solutions that possess one-half magnetic monopole charge.

The SU(2) YMH Lagrangian considered is $\mathscr{L}=-\frac{1}{4} F_{\mu \nu}^{a} F^{a \mu v}-\frac{1}{2} D^{\mu} \Phi^{a} D_{\mu} \Phi^{a}-\frac{1}{4} \lambda\left(\Phi^{a} \Phi^{a}-\right.$ $\left.\frac{\mu^{2}}{\lambda}\right)^{2}$, where $\mu$ is the Higgs field mass and $\lambda$ is the strength of the Higgs potential. The vacuum expectation value of the Higgs field is $\xi=\mu / \sqrt{\lambda}$. The covariant derivative of the Higgs field is $D_{\mu} \Phi^{a}=\partial_{\mu} \Phi^{a}+g \varepsilon^{a b c} A_{\mu}^{b} \Phi^{c}$ and the gauge field strength is $F_{\mu \nu}^{a}=\partial_{\mu} A_{v}^{a}-\partial_{\nu} A_{\mu}^{a}+g \varepsilon^{a b c} A_{\mu}^{b} A_{\nu}^{c}$, where $g$ is the gauge field coupling constant. The metric used is $g_{\mu v}=(-+++)$. The $\mathrm{SU}(2)$ internal group indices $a, b, c=1,2,3$ and the space-time indices are $\mu, v, \alpha=0,1,2,3$ in Minkowski space. The equations of motion that follow from the Lagrangian are

$$
D^{\mu} F_{\mu \nu}^{a}=\partial^{\mu} F_{\mu \nu}^{a}+g \varepsilon^{a b c} A^{b \mu} F_{\mu \nu}^{c}=g \varepsilon^{a b c} \Phi^{b} D_{\nu} \Phi^{c}, D^{\mu} D_{\mu} \Phi^{a}=\lambda \Phi^{a}\left(\Phi^{b} \Phi^{b}-\xi^{2}\right) .
$$

In the limit of vanishing $\mu$ and $\lambda$, but non-vanishing expectation value, where $\xi=\mu / \sqrt{\lambda}$ tends to a nonzero constant, the Higgs potential vanishes and self-dual solutions can be obtained by solving the first order partial differential Bogomol'nyi equation, $B_{i}^{a} \pm D_{i} \Phi^{a}=0$, where $B_{i}^{a}=-\frac{1}{2} \varepsilon_{i j k} F_{j k}^{a}$. The Abelian electromagnetic field suggested by 't Hooft [1] upon symmetry breaking is $F_{\mu \nu}=$ $\hat{\Phi}^{a} F_{\mu \nu}^{a}-\frac{1}{g} \varepsilon^{a b c} \hat{\Phi}^{a} D_{\mu} \hat{\Phi}^{b} D_{\nu} \hat{\Phi}^{c}=G_{\mu \nu}+H_{\mu \nu}$, where $G_{\mu \nu}=\partial_{\mu} A_{\nu}-\partial_{\nu} A_{\mu}$, is the gauge part and $H_{\mu \nu}=-\frac{1}{g} \varepsilon^{a b c} \hat{\Phi}^{a} \partial_{\mu} \hat{\Phi}^{b} \partial_{\nu} \hat{\Phi}^{c}$, is the Higgs part of the electromagnetic field. Here $A_{\mu}=\hat{\Phi}^{a} A_{\mu}^{a}$, the Higgs unit vector, $\hat{\Phi}^{a}=\Phi^{a} /|\Phi|$, and the Higgs field modulus $|\Phi|=\sqrt{\Phi^{a} \Phi^{a}}$.

The axially symmetric magnetic ansatz [4] with $\theta$-winding number $m=1$ and $\phi$-winding number $n=1$ used for our numerical construction of the one-half monopole is

$$
\begin{aligned}
& g A_{i}^{a}=-\frac{1}{r} \psi_{1}(r, \theta) \hat{n}_{\phi}^{a} \hat{\theta}_{i}+\frac{1}{r \sin \theta} P_{1}(r, \theta) \hat{n}_{\theta}^{a} \hat{\phi}_{i}+\frac{1}{r} R_{1}(r, \theta) \hat{n}_{\phi}^{a} \hat{r}_{i}-\frac{1}{r \sin \theta} P_{2}(r, \theta) \hat{n}_{r}^{a} \hat{\phi}_{i}, \\
& g A_{0}^{a}=0, \quad g \Phi^{a}=\Phi_{1}(r, \theta) \hat{n}_{r}^{a}+\Phi_{2}(r, \theta) \hat{n}_{\theta}^{a},
\end{aligned}
$$

where $P_{1}(r, \theta)=\sin \theta \psi_{2}(r, \theta), P_{2}(r, \theta)=\sin \theta R_{2}(r, \theta)$. Here $\psi_{1}(r, \theta), \psi_{2}(r, \theta), R_{1}(r, \theta)$, and $R_{2}(r, \theta)$ are the axially symmetric profile functions of the gauge potential $A_{i}^{a}$ and the isospin unit vectors are $\hat{n}_{r}^{a}=\sin \theta \cos \phi \delta_{1}^{a}+\sin \theta \sin \phi \delta_{2}^{a}+\cos \theta \delta_{3}^{a}, \hat{n}_{\theta}^{a}=\cos \theta \cos \phi \delta_{1}^{a}+\cos \theta \sin \phi \delta_{2}^{a}-$ $\sin \theta \delta_{3}^{a}, \hat{n}_{\phi}^{a}=-\sin \phi \delta_{1}^{a}+\cos \phi \delta_{2}^{a}$. In the rectangular coordinate system, the Higgs unit vector 
is $\hat{\Phi}^{a}=\sin \alpha \cos \phi \delta^{a 1}+\sin \alpha \sin \phi \delta^{a 2}+\cos \alpha \delta^{a 3}$, where $\cos \alpha=\frac{\Phi_{1}}{|\Phi|} \cos \theta-\frac{\Phi_{2}}{|\Phi|} \sin \theta$. The 't Hooft magnetic field is then reduced to $B_{i}=B_{i}^{G}+B_{i}^{H}=-\varepsilon_{i j k} \partial_{j} \mathscr{A}_{k}$ where the gauge part, $g B_{i}^{G}=$ $-n \varepsilon_{i j k} \partial_{j} \cos \kappa \partial_{k} \phi$, and the Higgs part, $g B_{i}^{H}=-n \varepsilon_{i j k} \partial^{j} \cos \alpha \partial^{k} \phi$, with $\cos \kappa=\frac{\Phi_{2}}{|\Phi|} P_{1}-\frac{\Phi_{1}}{|\Phi|} P_{2}$. Here $\mathscr{A}_{i}$ is the 't Hooft's gauge potential. The magnetic field lines of the configuration is plotted by drawing the contour lines of $(\cos \alpha+\cos \kappa)=$ constant on the vertical plane $\phi=0$.

\section{The Numerical Construction of the One-Half Monopole}

The numerical finite energy one-half monopole solutions here are constructed by using the four exact one-half monopole solutions, the Type $A 1, A 2, B 1$, and $B 2$ of Teh et al. [5] as asymptotic solutions at large $r$ and connecting them numerically to the YMH trivial vacuum at small $r$. The exact asymptotic Type $A 1$ one-half monopole solution at $r=\infty$ is given by [5]

$$
\begin{aligned}
& P_{1}=\sin \theta-\frac{1}{2} \sin \frac{1}{2} \theta(1+\cos \theta), P_{2}=\cos \theta-\frac{1}{2} \cos \frac{1}{2} \theta(1+\cos \theta), \\
& \psi_{1}=\frac{1}{2}, R_{1}=0, \Phi_{1}=\xi \cos \frac{1}{2} \theta, \Phi_{2}=-\xi \sin \frac{1}{2} \theta .
\end{aligned}
$$

Since the magnetic ansatz (1.2) is form invariant under the gauge transformation $\omega=\exp \left(\frac{i}{2} \sigma^{a} \hat{n}_{\phi}^{a} f(r, \theta)\right)$, where $\sigma^{a}$ are the Pauli matrices, we can generate the other three solutions from the transformation,

$$
\begin{aligned}
& \psi_{1}^{\prime}=\left\{\psi_{1}-\partial_{\theta} f\right\}, P_{1}^{\prime}=\left\{P_{1} \cos f+P_{2} \sin f+n[\sin \theta-\sin (f+\theta)]\right\}, \\
& R_{1}^{\prime}=\left\{R_{1}+r \partial_{r} f\right\}, P_{2}^{\prime}=\left\{P_{2} \cos f-P_{1} \sin f+n[\cos \theta-\cos (f+\theta)]\right\}, \\
& \Phi_{1}^{\prime}=\left(\Phi_{1} \cos f+\Phi_{2} \sin f\right), \quad \Phi_{2}^{\prime}=\left(\Phi_{2} \cos f-\Phi_{1} \sin f\right),
\end{aligned}
$$

where $f_{\{A 1 \rightarrow A 2\}}=\theta, f_{\{A 1 \rightarrow B 1\}}=-\frac{\pi}{2}$, and $f_{\{A 1 \rightarrow B 2\}}=\theta+\frac{\pi}{2}$, will generate the Type $A 2, B 1$, and $B 2$ solutions respectively. We also note that $f_{\{A 2 \rightarrow B 2\}}=\frac{\pi}{2}, f_{\{B 1 \rightarrow B 2\}}=\theta+\pi$, and $f_{\{B 1 \rightarrow A 2\}}=\theta+\frac{\pi}{2}$. Hence the transformations between the Type 1 and 2 solutions are non trivial transformations and they exist in different topological sectors. These solutions possess a magnetic charge, $\frac{1}{2 g}$, at $r=0$, gauge potential, $\mathscr{A}_{i}=\left\{\frac{\cos \theta \pm 1}{2 r \sin \theta}\right\} \hat{\phi}_{i}$, and magnetic fields, $g B_{i}=\frac{\hat{r}_{i}}{2 r^{2}} \mp 2 \pi \delta\left(x_{1}\right) \delta\left(x_{2}\right) \theta\left( \pm x_{3}\right) \delta_{i}^{3}$, for the Type $A$ and $B$ solutions respectively. They therefore carry a positive one-half monopole at the origin and a semi-infinite Dirac string which is a delta function line singularity of magnetic flux $\frac{2 \pi}{g}$ going into the origin. Hence the net magnetic charge of the configuration is zero.

The magnetic ansatz (1.2) reduces the equations of motion (1.1) to six coupled nonlinear second order partial differential equations which are solved asymptotically first at small and then at large distances. The numerical one-half monopole solutions are constructed by joining the exact asymptotic solutions at large distances (2.1) - (2.2) to the trivial vacuum solution at small $r$ and by fixing the boundary conditions for all the profile functions (1.2) along the $z$-axis and near the origin. Since the function $R_{2}(r, \theta)$ is singular along one-half of the $z$-axis, we choose to perform our numerical analysis with the functions, $P_{1}(r, \theta)$, and $P_{2}(r, \theta)$.

Near the origin, we have the common trivial vacuum solution for all the four solutions. The asymptotic solutions and boundary conditions at small distances that will give rise to finite energy one-half monopole solutions are

$$
\begin{array}{r}
\psi_{1}=P_{1}=R_{1}=P_{2}=0, \quad \Phi_{1}=\xi_{0} \cos \theta, \quad \Phi_{2}=-\xi_{0} \sin \theta \\
\sin \theta \Phi_{1}(0, \theta)+\cos \theta \Phi_{2}(0, \theta)=0,\left.\partial_{r}\left(\cos \theta \Phi_{1}(r, \theta)-\sin \theta \Phi_{2}(r, \theta)\right)\right|_{r=0}=0 .
\end{array}
$$


The boundary conditions imposed along the positive (negative) and negative (positive) $z$-axis for the profile functions (1.2) of the Type $A(B)$ solutions are

$$
\begin{array}{r}
\left.\partial_{\theta} \Phi_{1}(r, \theta)\right|_{\theta=0(\pi)}=\left.\Phi_{2}(r, \theta)\right|_{\theta=0(\pi)}=\left.\partial_{\theta} \psi_{1}(r, \theta)\right|_{\theta=0(\pi)}=0, \\
\left.R_{1}(r, \theta)\right|_{\theta=0(\pi)}=\left.P_{1}(r, \theta)\right|_{\theta=0(\pi)}=\left.P_{2}(r, \theta)\right|_{\theta=0(\pi)}=0, \\
\left.\Phi_{1}(r, \theta)\right|_{\theta=\pi(0)}=\left.\partial_{\theta} \Phi_{2}(r, \theta)\right|_{\theta=\pi(0)}=\left.\partial_{\theta} \psi_{1}(r, \theta)\right|_{\theta=\pi(0)}=0, \\
\left.R_{1}(r, \theta)\right|_{\theta=\pi(0)}=\left.P_{1}(r, \theta)\right|_{\theta=\pi(0)}=\left.\partial_{\theta} P_{2}(r, \theta)\right|_{\theta=\pi(0)}=0 .
\end{array}
$$

In our numerical calculation we set $\xi=g=1$. The numerical one-half monopole solutions connecting the asymptotic solutions (2.1) - (2.2) at large distances to the trivial vacuum solution (2.3) at small distances and subjected to the boundary conditions (2.4), and (2.5) together with the gauge fixing condition, [4] $r \partial_{r} R_{1}-\partial_{\theta} \psi_{1}=0$, are solved using Maple and MATLAB [7]. The reduced six partial differential equations of motion are then transformed into a system of nonlinear equations using the finite difference approximation. The numerical calculations are performed with the system of nonlinear equations been discretized on a non-equidistant grid of size $90 \times 80$ covering the integration regions $0 \leq \bar{x} \leq 1$ and $0 \leq \theta \leq \pi$. Here $\bar{x}=\frac{r}{r+1}$ is the finite interval compactified coordinate. The partial derivative with respect to the radial coordinate is then replaced accordingly by $\partial_{r} \rightarrow(1-\bar{x})^{2} \partial_{\bar{x}}$ and $\frac{\partial^{2}}{\partial r^{2}} \rightarrow(1-\bar{x})^{4} \frac{\partial^{2}}{\partial \bar{x}^{2}}-2(1-\bar{x})^{3} \frac{\partial}{\partial \bar{x}}$. We first used Maple to find the Jacobian sparsity pattern for the system of nonlinear equations. After that we provide this information to MATLAB to run the numerical computation. The system of nonlinear equations are then solved numerically using the trust-region-reflective algorithm by providing the solver with good initial guess. The second order equations of motion are solved when $0 \leq \lambda \leq 12$. For values of $\lambda$ exceeding 12 the numerical accuracy decreases. In our calculations, the overall error estimate is $10^{-4}$.

The numerical profile functions, $\psi_{1}, P_{1}, R_{1}, P_{2}, \Phi_{1}$, and $\Phi_{2}$ of all the four one-half monopole solutions are regular functions of $r$ and $\theta$. However the function $R_{2}=\frac{P_{2}}{\sin \theta}$ possesses a string singularity along the negative (positive) $z$-axis for the Type $A(B)$ solutions. The 't Hooft's gauge potential at large $r$, tends to $\mathscr{A}_{i}=\left.(\cos \alpha+\cos \kappa) \partial_{i} \phi\right|_{r \rightarrow \infty}=\frac{\hat{\phi}_{i}}{r \sin \theta}\left\{\frac{1}{2}(\cos \theta \pm 1)+\frac{F_{G}(\theta)}{r}\right\}$, where $F_{G}(\theta)=\left.r\left\{\frac{\Phi_{2}}{|\Phi|}\left(P_{1}-\sin \theta\right)-\frac{\Phi_{1}}{|\Phi|}\left(P_{2}-\cos \theta\right)-\frac{1}{2}(\cos \theta \pm 1)\right\}\right|_{r \rightarrow \infty}$. From the graphs of $F_{G}(\theta)$ versus angle $\theta$, we find that $F_{G}(\theta)=\mu_{m} \sin ^{2} \theta$, where the dimensionless magnetic dipole moment, $\mu_{m}$ is read from the graphs at $\theta=\frac{\pi}{2}$. The graphs of $\mu_{m 1}$ and $\mu_{m 2}$ of the Type $A$ solutions versus $\lambda^{1 / 2}$ are shown in Figure 1 (a) and their values are given in Table 1 . The magnetic moment, $\mu_{m}$, is positive for the Type $A$ solutions and negative for the Type $B$ solutions. We calculate numerically for the net magnetic charge $M=M_{G}+M_{H}$, and plot the graphs of $M, M_{G}$ and $M_{H}$ versus $\bar{x}$-axis as shown in Figure 1 (b). We note that we are only able to obtain the magnetic charge of the one-half monopole from the numerical data. The numerical data cannot account for the magnetic charge carried by the Dirac string. We also notice from the plots of Figure 1 (b) that $M=\frac{1}{2}$ when $r \geq 4$ and its value vanishes as $r$ tends to zero.

The total dimensionless energy, $E=\frac{g}{8 \pi \xi} \int\left\{B_{i}^{a} B_{i}^{a}+D_{i} \Phi^{a} D_{i} \Phi^{a}+\frac{\lambda}{2}\left(\Phi^{a} \Phi^{a}-\xi^{2}\right)^{2}\right\} d^{3} x$, when $\lambda=0$ is about two percent higher than the BPS value of $E_{B P S}=\frac{1}{2}$. The total energies $E_{1}$ and $E_{2}$ are plotted versus $\lambda^{1 / 2}$ as shown in Figure 1 (c) and their values are given in Table 1. The 3D surface and contour line plots of $\mathscr{E}$, the energy density weighted by a factor $r^{2} \sin \theta$ of the Type $A 1$ 


\begin{tabular}{|c|c|c|c|c|c|c|c|c|c|c|c|}
\hline$\lambda$ & 0 & 0.04 & 0.20 & 0.40 & 0.60 & 0.80 & 1.00 & 2.00 & 4.00 & 8.00 & 12.00 \\
\hline$\mu_{m 1}$ & 1.32 & 1.22 & 1.15 & 1.11 & 1.09 & 1.07 & 1.06 & 1.02 & 0.98 & 0.94 & 0.92 \\
\hline$\mu_{m 2}$ & 1.04 & 0.96 & 0.91 & 0.88 & 0.86 & 0.85 & 0.84 & 0.81 & 0.78 & 0.74 & 0.73 \\
\hline$E_{1}$ & 0.51 & 0.54 & 0.56 & 0.57 & 0.58 & 0.59 & 0.59 & 0.61 & 0.63 & 0.66 & 0.67 \\
\hline$E_{2}$ & 0.53 & 0.55 & 0.58 & 0.59 & 0.60 & 0.60 & 0.61 & 0.63 & 0.65 & 0.67 & 0.69 \\
\hline
\end{tabular}

Table 1: The magnetic dipole moments $\mu_{m 1}, \mu_{m 2}$, and total energies $E_{1}, E_{2}$, of the Type $A 1$ and $A 2$ solutions for $0 \leq \lambda \leq 12$.

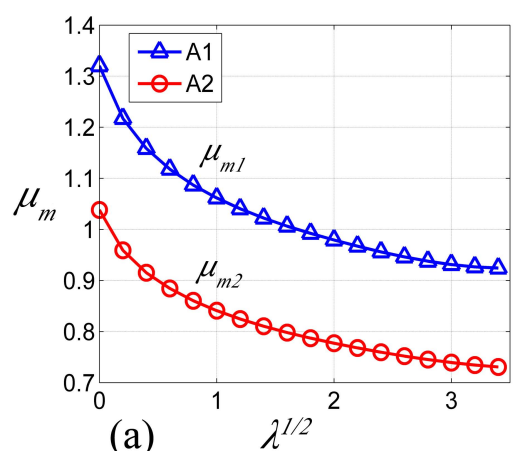

(a)

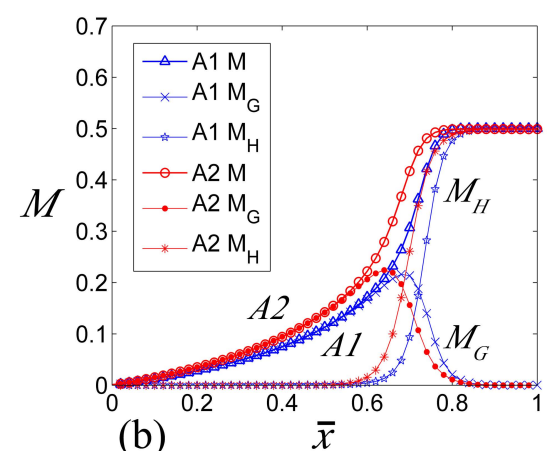

(b)

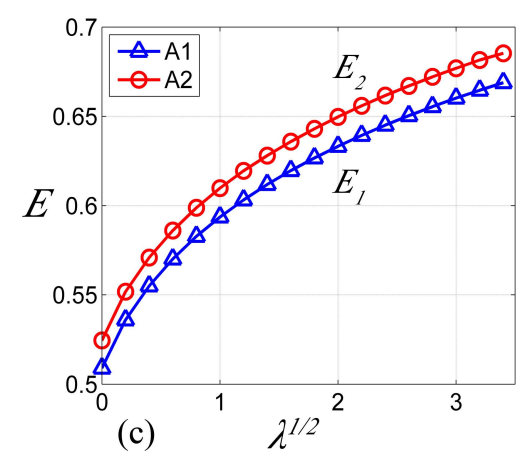

(c)

Figure 1: (a) Plot of the magnetic dipole moments $\mu_{m 1}$ and $\mu_{m 2}$ versus $\lambda^{1 / 2}$ for the Type $A$ solutions. (b) Plot of the magnetic charges $M, M_{G}$ and $M_{H}$ versus $\bar{x}$ when $\lambda=1$. (c) Plots of the total energies $E_{1}$ and $E_{2}$ versus $\lambda^{1 / 2}$.

solution along the $x-z$ plane at $y=0$ when $\lambda=1$ are shown in Figure 2 (a). The one-half monopole is shaped like a rugby ball (prolate spheriod) with its energy concentrated along the negative $z$-axis near $r=0$ for the Type $A$ solutions. The 3D surface and contour plots of the Higgs field modulus, $|\Phi|$, of the Type $A 1$ solution along the $x-z$ plane at $y=0$ when $\lambda=1$, show that there is a point zero of the Higgs field modulus at the origin, Figure 2 (b). Hence the one-half monopoles is located at $r=0$ where the Higgs field vanishes. The shape of the 3D surface plots for the one-half monopole is that of a flatten cone and there is a double zero of $|\Phi|$ at $r=0$ along the negative $z$-axis for the Type $A$ solutions. The magnetic field lines contour plot of the Type $A 1$ solution along the $x-z$ plane at $y=0$ is shown in Figure 2 (c) when $\lambda=1$.

\section{Remarks}

From our analysis, we conclude that the Type $A$ and $B$ solutions are gauge equivalent as they are connected by constant gauge transformations, $f_{\{A 1(A 2) \rightarrow B 1(B 2)\}}=\mp \frac{\pi}{2}$. They are just a $180^{\circ}$ rotation of each other about the origin, $r=0$. The Type 1 and 2 solutions are connected by non trivial gauge transformations, $f_{\{A 1(B 1) \rightarrow A 2(B 2)\}}=\theta+0(\pi)$, and they exist in different topological sectors of the YMH theory. Theoretically, they should possess the same gauge invariant quantities. Our numerical analysis however shows that they possess slightly different (about three percent) total energies and magnetic dipole moments. We believe that the differences are due to the lack of symmetry of the large $r$ exact asymptotic solutions in the $m=1$ gauge. We have repeated our numerical constructions of the one-half monopole solutions in the $m=+\frac{1}{2}$ (Type 1) and $m=$ 


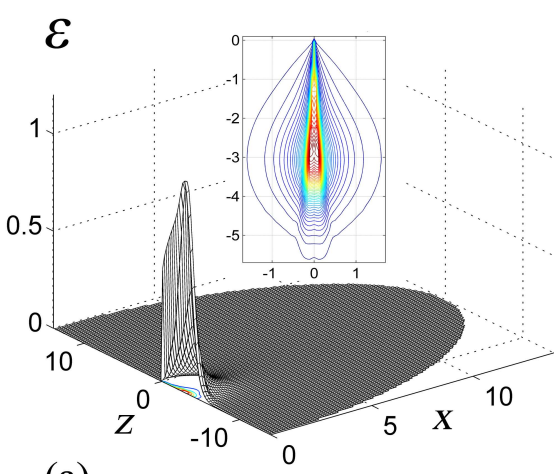

(a)

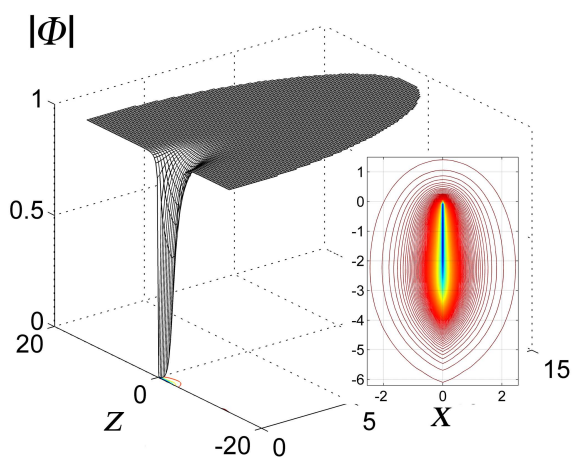

(b)

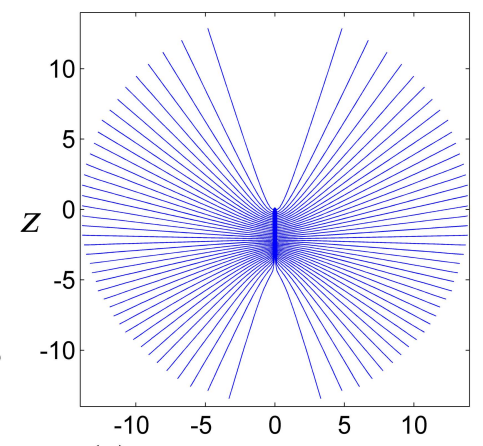

(c)

Figure 2: 3D surface and contour (inset) plots of (a) the weighted energy density, $\mathscr{E}$, and (b) the Higgs field modulus, $|\Phi|$. (c) The contour plot of the magnetic field lines. The plots here are for the Type $A 1$ solution along the $x$-z plane at $y=0$ when $\lambda=1$.

$-\frac{1}{2}$ (Type 2) gauges and found that the differences between $E_{1}$ and $E_{2}$ and between $\mu_{1}$ and $\mu_{2}$ are negligible. This is because in the $m= \pm \frac{1}{2}$ gauges the large $r$ exact asymptotic solutions are symmetrical [8] and the numerical accuracy of both Type 1 and 2 solutions are the same. Hence the Type 1 and 2 solutions are one-half monopole existing in different topological sectors of the YMH theory.

The authors would like to thank Universiti Sains Malaysia for the RU research grant (account number: 1001/PFIZIK/811180).

\section{References}

[1] G. 't Hooft, Nucl. Phy. B79, 276 (1974); A.M. Polyakov, Sov. Phys. - JETP 41, 988 (1975); Phys. Lett. B59, 82 (1975); JETP Lett. 20, 194 (1974).

[2] M.K. Prasad and C.M. Sommerfield, Phys. Rev. Lett. 35, 760 (1975); E.B. Bogomol'nyi, Sov. J. Nucl. Phys. 24, 449 (1976); E.B. Bogomol'nyi and M.S. Marinov, Sov. J. Nucl. Phys. 23, 355 (1976).

[3] C. Rebbi and P. Rossi, Phys. Rev. D22, 2010 (1980); R.S. Ward, Commun. Math. Phys. 79, 317 (1981); P. Forgacs, Z. Horvarth and L. Palla, Phys. Lett. B99, 232 (1981); Nucl. Phys. B192, 141 (1981); M.K. Prasad, Commun. Math. Phys. 80, 137 (1981); M.K. Prasad and P. Rossi, Phys. Rev. D24, 2182 (1981).

[4] B. Kleihaus and J. Kunz, Phys. Rev. D61, 025003 (2000); B. Kleihaus, J. Kunz, and Y. Shnir, Phys. Lett. B570, 237, (2003); B. Kleihaus, J. Kunz, and Y. Shnir, Phys. Rev. D68, 101701 (2003); Phys. Rev. D70, 065010 (2004).

[5] Rosy Teh, K.G. Lim and P.W. Koh, FRONTIERS IN PHYSICS: 3rd International Meeting, Kuala Lumpur (Malaysia), 12-16 January 2009, edited by S.P. Chia, M.R. Muhammad, and K. Ratnavelu, ISBN: 978-0-7354-0687-2, AIP Conference Proceedings Volume 1150, 424 (2009).

[6] E. Harikumar, I. Mitra, and H.S. Sharatchandra, Phys. Lett. B557, 303 (2003).

[7] K.G. Lim, Rosy Teh and K.M. Wong, J. Phys. G: Nucl. Part. Phys. 39, 025002 (2012).

[8] Rosy Teh, B.L. Ng, and K.M. Wong, The Twin Effect of the SU(2) Yang-Mills-Higgs Monopole Solutions, 2012 National Conference in Physics, PERFIK 2012, Colmar Tropicale, Bukit Tinggi, Pahang, Malaysia, November 19 - 21, 2012; AIP Conf. Proc. 1528, 201 (2013). 